\title{
X-RAY AND GAMMA-RAY OBSERVATIONS OF ISOLATED NEUTRON STARS
}

\author{
D.J. THOMPSON \\ Laboratory for High Energy Astrophysics \\ NASA Goddard Space Flight Center \\ Greenbelt, Maryland USA
}

\section{Introduction}

Pulsars provide useful diagnostics of isolated neutron stars, because the timing information allows many physical parameters to be derived. This brief review describes some of the X-ray and gamma-ray properties of pulsars.

\section{Light Curves}

As shown in Figure 1, pulsar light curves at high energies are varied in shape. The preponderance of double pulses with a bridge of emission suggests emission from a cone or surface above a single pole of the neutron star. Very young pulsars (Crab, PSR B0540-69, PSR B1509-58) and at least one old, ms pulsar (PSR B1821-24) show only magnetospheric emission associated with accelerated charged particles, as indicated by the similarity of the pulse shape across the entire X-ray and gamma-ray band. Intermediateage pulsars (Vela, Geminga, PSR B0656+14, PSR B1055-52) also reveal a thermal component in soft X-rays, probably from near the surface of the star. This component differs in both shape and phase from the higherenergy, nonthermal radiation.

\section{Broadband Energy Spectra}

Figure 2 shows the energy spectra of several pulsars across the entire electromagnetic spectrum. In all cases the observable output is dominated by the $\mathrm{X}$-ray and gamma-ray emission, indicating the importance of these highenergy radiations for pulsars. For the youngest and oldest pulsars, repre- 
sented here by the Crab (age about $1000 \mathrm{y}$ ) and PSR B1821-24 (age greater than $10^{7} \mathrm{y}$ ), the emission peaks in the hard X-ray band. The intermediateage pulsars (Vela has a timing age of about $1 \times 10^{4} \mathrm{y}$; Geminga's age is about $3 \times 10^{5} \mathrm{y}$ ) are seen most prominently in the high-energy gamma-ray range. All spectra have some high-energy cutoff.

\section{Trends and Models}

The energy spectra of Figure 2, integrated over energy, can be used along with the distance estimates from radio and optical observations to construct a broadband luminosity L for these high-energy pulsars. A trend is seen for $\mathrm{L}$ to be linearly proportional to $\dot{E}^{1 / 2}$, the spin-down luminosity. This quantity is also proportional to the open-field-line voltage or the polar cap current (Harding, 1981). The broadband luminosity trend contrasts with the trend for X-rays alone, which shows a linear dependence on $\dot{E}$ (Becker and Trümper, 1997). As shown by Goldoni and Musso (1996), other possible phenomenological trends are less convincing.

Both polar-cap and outer-gap models have evolved with the discoveries of new X-ray and gamma-ray pulsars. Although both types can explain many observed features, neither appears to offer a complete enough picture to warrant broad generalizations. For recent summaries, see Daugherty and Harding (1996) and Romani (1996). Future observations, from operating telescopes such as ASCA and RXTE, and from future telescopes such as AXAF, XMM and GLAST, should resolve many of the open questions.

\section{References}

Becker, W., and Aschenbach, B. 1995, in The Lives of Neutron Stars, (eds A. Alpar, U. Kiziloğlu \& J. van Paradijs), Kluwer Academic Publishers, p. 47.

Becker, W., \& Trümper, J. 1997, $A \& A p$, in press

Daugherty, J. K. \& Harding, A. K. 1996, $A p J$, 458, 278.

Goldoni, P, \& Musso, C. 1996, $A \& A p S, 120,103$.

Halpern, J.P. \& Wang, F. Y.-H. 1997, ApJ, 477, 905.

Harding, A.K. 1981, ApJ, 245, 267.

Kanbach, G. 1997, Proceedings of NATO Advanced Study Institute, The Many Faces of Neutron Stars, Lipari, October, 1996, in press

Kuiper, L. et al. 1997a, in Proceedings of NATO Advanced Study Institute, The Many Faces of Neutron Stars, Lipari, October, 1996, in press

Kuzmin, A.D. \& Losovsky, B. Y. 1997, IAU Circ. 6559

Much, R. et al. 1997, Proc. 4th Compton Symposium, (ed. C. Dermer and M. Strickman), AIP, in press

Nel, H.I. et al. 1996, $A p J, \mathbf{4 6 5}, 898$

Romani, R.W. 1996, $A p J, 470,469$

Saito, Y. et al. 1997, $A p J$, in press

Shitov, Yu. P. \& Pugachev, V.D. 1997, preprint

Thompson, D.J. 1996, in Pulsars: Problems and Progress, IAU Colloquium 160 (ed. M. Bailes, S. Johnston, M. Walker) 


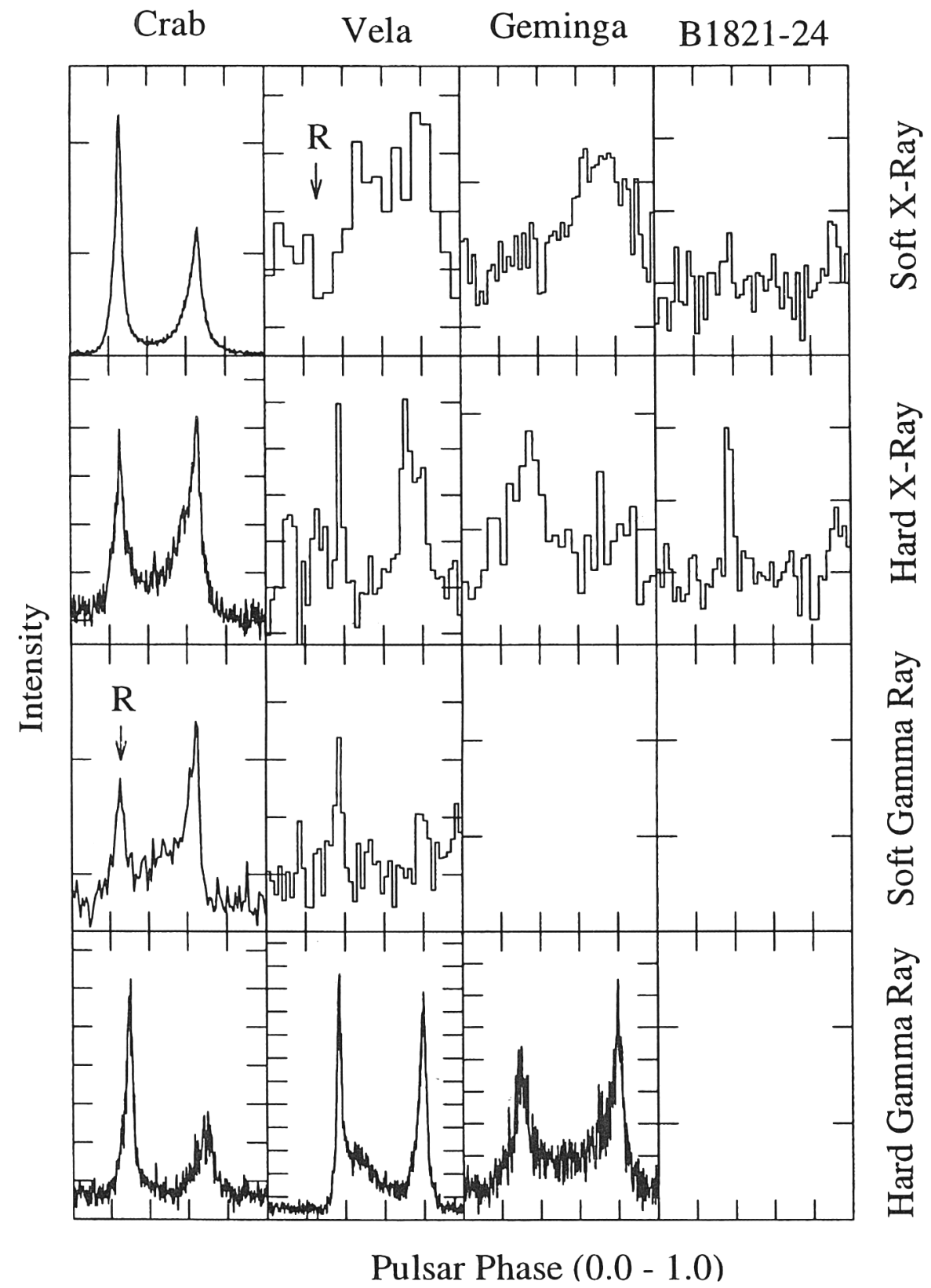

Figure 1. Light curves of four pulsars at X-ray and gamma-ray energies, updated from Thompson (1996). New references: Crab: Becker and Aschenbach (1995), Much et al. (1997), Kanbach (1997); Vela: Harding and Strickman, priv. com., Kuiper et al. (1997); PSR B1821-24: Saito et al. (1997) 


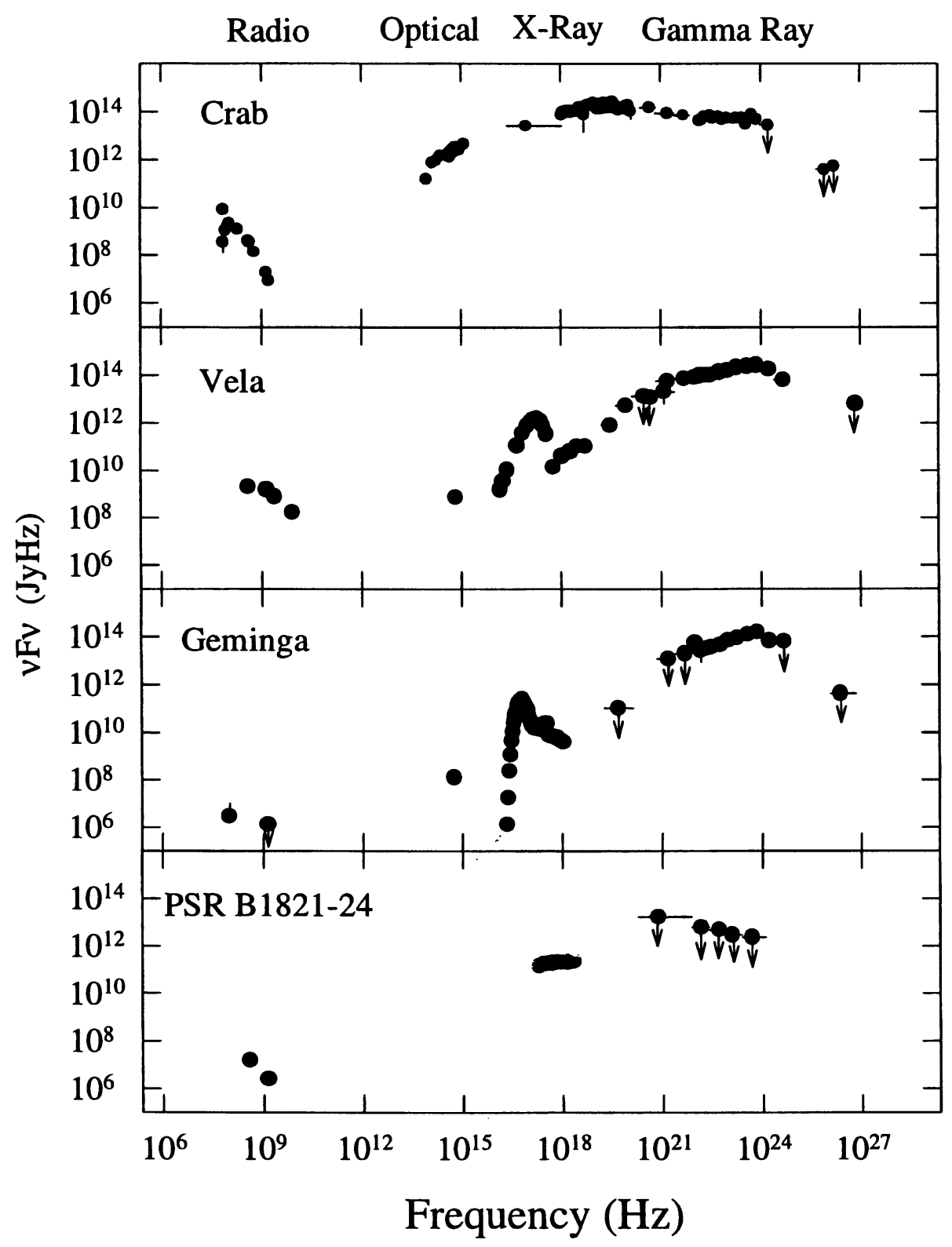

Figure 2. Energy spectra of four pulsars, updated from Thompson (1996). New references: Geminga: Kuzmin and Losovsky (1997), Shitov and Pugachev (1997), Halpern and Wang (1997), Vela: Harding and Strickman, priv. com., Kuiper et al. (1997); PSR B1821-24: Saito et al. (1997), Nel et al. (1996) 